Please do not remove this page

RMIT

UNIVERSITY

\title{
Displaced mothers: Birth and resettlement, gratitude and complaint
}

Niner, Sara; Kokanovic, Renata; Cuthbert, Denise

https://researchrepository.rmit.edu.au/esploro/outputs/9921859143101341/filesAndLinks?institution=61RMIT_INST\&index=null

Niner, S., Kokanovic, R., \& Cuthbert, D. (2013). Displaced mothers: Birth and resettlement, gratitude and complaint. Medical Anthropology: Cross-Cultural Studies in Health and Illness, 32(6), 535-551. https://doi.org/10.1080/01459740.2013.769103

Document Version: Accepted Manuscript

Published Version: https://doi.org/10.1080/01459740.2013.769103

Repository homepage: https://researchrepository.rmit.edu.au

(C) 2013 Taylor \& Francis Group, LLC

Downloaded On 2023/04/27 00:10:37 +1000

Please do not remove this page 
Thank you for downloading this document from the RMIT Research Repository.

The RMIT Research Repository is an open access database showcasing the research outputs of RMIT University researchers.

RMIT Research Repository: http://researchbank.rmit.edu.au/

\section{Citation:}

Niner, S, Kokanovic, R and Cuthbert, D 2013, 'Displaced mothers: Birth and resettlement, gratitude and complaint', Medical Anthropology: Cross-Cultural Studies in Health and Illness, vol. 32, no. 6, pp. 535-551.

See this record in the RMIT Research Repository at:

https://researchbank.rmit.edu.au/view/rmit:23090

Version: Accepted Manuscript

Copyright Statement: (c) 2013 Taylor \& Francis Group, LLC

Link to Published Version:

http://dx.doi.org/10.1080/01459740.2013.769103 
Displaced mothers: birth and resettlement-gratitude and complaint ( 8600 words)

\section{Abstract: 150 words}

In this article we explore birthing experiences of displaced Karen women from Burma both before and after resettlement in Australia. Women's narratives were framed by experiences of persecution and displacement. Although grateful for the security of resettlement in Australia, social inclusion was negligible and women’s birthing experiences occurred in that context. Women described the impact of the lack of interpreting services in Australian hospitals and an absence of personal and communal care that they expected. Frequently, this made straightforward births confusing or difficult, and exacerbated the distress of more complicated births. Differences in individual responses related to women’s histories, with younger women displaying more preparedness to complain and identify discrimination. The problems identified with health care coupled with the inability of many of the women to complain await address not just within the health-care system, but more widely as part of social attitudes concerning Australia’s obligations to those who seek asylum.

Keywords: perinatal healthcare, refugee, immigrant, resettlement, disparity 25 word media line: Challenges experienced by forced migrant women birthing in Australia await address within health-care systems and more widely as part of Australia’s obligations to asylum seekers. 


\section{Introduction}

Contemporary global transformations have created cultural shifts and population flows of displaced peoples and forced migrants. In this article we consider the accounts of some women who have been subject to these flows, those 'who have suffered the sentence of history—subjugation, domination, diaspora, displacement' (Bhabha 1994, 246). Significant rises in xenophobia and intolerance accompany these transformations and population flows (Quesada, Kain Hart and Bourgois 2012, 894). Displaced and forced migrants are particularly vulnerable to the inequalities inherent in international flows of 'income, investment, education and other social resources' within a 'hegemonic gender order of post-colonial states' (Connell 2012). This article examines some effects of inequality consequent on these flows through a focus on the birthing experiences of forced migrant women in Australian hospitals.

The tendency toward the legal and bureaucratic institutionalization of intolerance determines 'deservingness' to use social services such as hospitals. The assumptions that underlie various migrants' entitlement to the provision of health care in resettlement is debated in a growing international discourse (see Grove and Zwi 2006; Medical Anthropology (Special Issues), 2011 30(4, 5); Social Science and Medicine (Special Issue), 2012 74(6); Viruell-Fuentes, Mirada and Abdulrahmin 2012). Popular and political understandings of immigrants' 'deservingness' shape and reflect formal policies and legal 'entitlements' (Sargent 2012, 855-857). The 'deservingness' discourse may impact on migrants' sense of self-worth, 'as some convince themselves they are undeserving or unworthy of health care or are undesirable political subjects' (Quesada 2012, 896). Some immigrants do not claim a right to health care or assert a sense of entitlement more broadly thus ‘internalizing ... a socially devalued identity that is generated by anti-immigrant discourse' (Sargent 2012, 856). We note that such 
identities may precede resettlement and emerge from initial displacement through experiences of discrimination, persecution and marginalization. While the majority of these debates are set in the US where health care provision is vexed and complicated by large numbers of illegal migrants these broad trends are relevant to Australia and we hope to demonstrate this in the local narratives of forced migrants analysed here.

The rise in intolerance affecting displaced peoples is linked to processes of 'othering' which serve to ‘mark and name’ difference (Grove and Zwi 2006). Grove and Zwi (2006) locate barriers to health care for forced migrants in Australia within a wider process of 'othering', contending that forced migrants are increasingly represented as threats to 'community and sovereignty'. Many Australians hold negative views about forced migrants (referred to as refugees or asylum seekers) because of perceived, ‘undesirable individual behaviours' and threats posed to Australian values and culture from migrants who maintain their own languages and customs (McKay, Thomas and Kneebone 2011). Many believe migrants seek personal benefit and exploit welfare systems (McKay, Thomas and Kneebone 2011). Futher the granting of asylum or safe haven is often understood as an act of humanitarianism or charity rather than as Australia's international legal obligation to which displaced peoples have a human right. This logic can trap forced migrants in a state of 'gracious acceptance' which silences or hinders the assertions of rights or complaints about treatment or services in their host society (cf. Grove and Zwi 2006, 1933-5). We trace similar reactions here but are mindful of women's own agency in this regard and the variety and individuality of their responses which are described in this paper.

Long-standing debate focuses on migrant access to healthcare in Australia (Manderson and Allotey 2003). This debate highlights migrants’ capacity to use health services and refers to 'barriers' to access from lack of knowledge of health 
frameworks and services, lack of transport or language and communication skills. The combination of individual limitations and health-care provider barriers are factors.

The 'barriers' explanation tends to place responsibility on migrants for their 'lack' of acculturation and shifts blame for poor health outcomes onto the culture of the patient. (Kokanovic et al 2008 and 2010; Kokanovic and Stone 2010; Viruell-Fuentes, Mirada and Abdulrahmin 2012). Yet recognition of the limitations of migrants to use health services does not always result in blaming them for their own poor health outcomes, rather it may simply illuminate the need for recipient societies to provide more preparation, support, and advocacy for migrants.

The complications faced by ethnic minorities in using health services are intricately drawn in one case study of the Hmong in the US by Fadiman (1997) who traces the case of a Hmong child whose health care was compromised by an impassable cultural gulf or 'cultural collision', between the Hmong and the American health care providers. Fadiman demonstrates the consequences of blocked communication in critical health care processes.

Dossa (2002) identifies a 'cultural-barriers-to-health-care paradigm' which provides the framework of much research in this field. Within this paradigm, most research is geared towards recommendations to remove 'barriers' and improve access to health services rather than reflecting an deeper appreciation of the situation of users or their understandings and much less reflecting back on the politics of health care and why it could not accommodate users initially. Nor is this kind of research particularly 'mindful' of its ‘own sociocultural, economic and historical location' (Anderson et al 2003, 196). This broader trajectory outlined in the international literature shifts the focus away from the culture of the patient, any limitations to use health services and the barriers they may face and onto 'the culture of the health provider and society that 
it operates within and the power dimension of race, class, gender and immigrant status and how these shape health inequities’ (Viruell-Fuentes, Mirada and Abdulrahmin 2012, 2). We return to this point in the conclusion.

Recommendations to overcome disparities in health care for migrants, and also indigenous peoples, have included transcultural nursing, cultural safety and competency approaches (Anderson et al 2003). Laura Griffith (2010, 290) argues instead for: '...a health service that can respond to the complexity of individual narratives and produce reflexive practitioners who move beyond epidemiological or cultural categories'. Anderson also shifts the focus away from cultural barriers to the power relations inherent in health care exchanges:

Rather than focusing on exotic belief systems of people from different ethnocultural backgrounds, and treating each group as a distinct entity, we are challenged ... to examine the unequal relations of power that are the legacy of the colonial past and neocolonial present, and the ways in which the cultures of dominant groups have redefined local meanings, and dictated social structures, including health care delivery systems. (Anderson et al 2003, 196-197)

Griffith's adds that it is imperative that 'narratives of the women themselves are reflexively analyzed to see in which ways they contest, or align themselves with, the prevailing institutionalized narratives of their experience' (Griffith 2010, 290). This is an important initiative and this article provides a critical and reflexive analysis of the narratives of the birthing experiences of Karen women in Australia. Further work however is still required on how those narratives contest or align themselves with 'institutionalized’ narratives including the perspectives of health-care providers. 


\section{The study}

The qualitative study from which the analysis in the article is derived examined the effects of displacement on the lives of a group of ethnic minority Karen women from Burma-Myanmar through a focus on perinatal health. Data is drawn from the accounts of birth and perinatal emotional health of 15 Karen women, now resettled in Australia (Kokanovic et al 2012). Burma faces ongoing conflict resulting from the end of British colonial rule in 1948. The breakdown of ethno-political relations led to armed conflict between the central Burmese state and ethnic minorities such as the Karen (O’Kane 2005, 7; Oo and Kusakabe 2010). Participants and their families fled persecution and armed attacks by the State army, followed by displacement and ongoing persecution within Burma and eventual marginalization in refugee camps. Their narratives document lifetimes of discrimination and alterity: as ethnic minority peoples in opposition, as displaced persons in Thailand, and as forced migrants to a foreign culture, which in many regards has been ill-prepared to accommodate them. Their lives have been shaped by global political flows that they embody at local levels. Women who had recently given birth in Australia were purposefully selected through recruitment by several Karen community networks in Australia. Fifteen women in all volunteered and engaged in interviews with researchers. A key aim was to understand how pregnancy and birth was experienced both before and after resettlement in Australia by forced migrant women. As perinatal distress is influenced by the broader contexts of women's lives the methodology used to capture this was narrative style ethnographic research (Kokanovic et al 2012). As women were generally unfamiliar with the concept of peri-natal distress two women, who has shown particular insight were selected for a second interview to ask more specific questions about cultural understandings of emotional health (17 interviews in total). A bi-lingual Karen 
Research Assistant who shared language and the experiences of forced migration with interviewees undertook the interviews. All participants chose to be interviewed in their first language, Karen which was important not only because of low levels of English proficiency but because language is known to be a powerful organizer of cognitive and affective experience (Tummala-Narra 2004, 169), particularly so with displaced Karen mothers (Oo and Kusakabe 2010).

A verbatim transcript in Karen was prepared and translated into English by the research assistant and then key themes, ideas and commonalities were discussed and cultural and background context added. Pseudonyms were either chosen by participants or assigned by the Research Assistant. Significant statements and phrases were identified within the narratives and categorized according to key themes and then findings were identified.

We then located findings within the framework of the contemporary literature on the health and illness experiences of displaced peoples or forced-migrants within western host societies. We found that the data resonated with debates surrounding 'deservingness' and this literature suggests reasons for why forced-migrants often encounter difficulties in utilizing health services.

A limitation of the data is the absence of perspectives of health care providers. We emphasize that our aim was to explore women's perspectives, which according to our literature review were largely absent. In this way, we aim to offer an emic perspective in anthropological terms but acknowledge that research synthesizing user and provider perspectives and experiences is needed.

The forced migrant is ‘structurally vulnerable’ to receiving poor health services. Evidence of 'intersectional data on perinatal health' demonstrates that this inequity 
extends to maternity care globally (Connell 2012, 1678). The women in this study have embodied these inequalities as will be presented below. Subjectively, this is acknowledged by the women in the relaying of an old Burmese adage, which describes women giving birth as being halfway between life and death. This precariousness was intensified by armed conflict and displacement and has not been fully resolved in resettlement.

Karen women's descriptions of their maternity care in Australian institutions need to be mediated by an understanding that their perspectives and emotional reactions have been shaped by often traumatic, past encounters of displacement. Their accounts must also be mediated by an appreciation of the disruptions and differences they have had to adapt to: from 'traditional' or pre-modern systems, to limited care in displaced persons camps and finally in western biomedical systems. Biomedical discourses and the 'corporations, professions and state agencies of the health sector' are gendered (Connell 2012, 1675) and one of the key manifestations of this gendering is the 'institutionalized control of women's reproductive capacities’ (Connell 2012, 1677). The initial encounter of the women in this study with first world health institutions was through obstetric care in Australian hospitals, which have among the highest rates of intervention into birth in the world (Donnellan-Fernandez 2010). These higher incidences 'in healthy women and babies is not associated with improved perinatal outcomes, but with increased risks for these mothers and babies (DonnellanFernandez 2010).

\section{Hearing women's voices}

This research addresses the absence of 'voices of marginalized and vulnerable groups' in medical anthropology debates (Sargent 2012, 857) in response to a key finding of 
the literature review for the original study that little research deals specifically with refugee-background women or frames their experiences within wider cultural and political contexts (Kokanovic et al 2012, 5). The oldest participant in the study was 47 and the youngest, 20. The largest family included eight children and three of the younger mothers had one child only. Children ranged in age from 21 years to one month. All women, except younger mother Rosy, were living with their husbands and father of their children, although several women mentioned martial discord. While younger mothers who are described below, did not mention personally experiencing violence and military attacks on natal villages in Karen state this may just mean they chose not to mention them during the interview. Further, such events were pervasive in their lives: existing in shared family histories and through long-term residence in refugee camps in close proximity to conflict zones. Several mentioned either attacks on camps or ongoing fear of such attacks. Activities in conflict zones were also mentioined: Hsa for instance, volunteered with a medical team who regularly crossed the border into conflict zones to treat those in need.

All participants had lived in displaced persons camps around the Thai border for extended periods, the younger women growing up in these environments, and had eventually received UN registration as refugees and arrived in Australia through associated legal humanitarian programs over the last five years. Similar to other displaced women’s narratives from the Thai-Burma border, responses were characterized by stories of surviving armed conflict (O’Kane 2005). Significant fear and trauma were associated with either personal or shared memories and experience or fear of bombardment, violence, loss and separation from family and the hardships of flight. Physical hardships rather than emotions were focused upon and concerns for 
emotional or mental health were rarely expressed (and then generally in spiritual terms).

Gratitude for safe haven in Australia was overt in some women’s accounts. Gratitude was directed toward Australian Government assistance programs, social services and improvements in their general life conditions since resettlement. Positive accounts of Australian life were tempered by the immediate challenges they faced in resettlement and the women's sense of loss of the people and communities they had left behind. Any sense of belonging and inclusion in Australian society were negligible in the accounts.

Further, gratitude for life in a secure environment where basic needs were met appeared in proportion to the levels of adversity and the absence of safety and care experienced by women prior to arrival to Australia, particularly in relation to birthing experiences. This appeared to influence reactions to treatment in Australian hospitals. Accounts ranged from those who were grateful for services in resettlement linked to a resigned acceptance of difficulties encountered to others more critical about services and several who identified discrimination. Younger women with better English language proficiency and understanding of modern systems appeared more able to critique services. The older women, who had born children in more difficult circumstances in Burma or Thailand, exhibited more appreciation of the care given by Australian services than the younger women, most of whom had not previously given birth. Age as a critical variable in processes of being able to engage with a new community and the psychological transition this entails has been noted with Karen communities in the US (Harkins 2012, 194).

In this article we discuss eight case studies from the 15 women interviewed to illustrate these two different responses indentified throughout all interviews. Baw, Say 
Say, Ruth and Ma Shah are four older mothers whose accounts resonate with the themes of 'normalising distress', self-reliance and a state of 'gracious acceptance' as outlined above. Nin, Wah, Rosy and Hsa represent the younger mothers whose accounts embody a stronger sense of entitlement reflected in their complaints about services.

However, our findings also reveal that within this broader patterning women who undergo similar experiences will have varied reactions to their circumstances. For example, some women appeared more vulnerable than others to emotional distress (such as Ma Shah or Nin’s more extreme reactions in comparison to Ruth or Rosy). These experiences are discussed more fully in the sections below and intervening factors discussed. Others followed 'imperatives to normalise distress' (Edge and Rogers 2005) (such as in the accounts of Say Say or Ruth below). Such imperatives along with efforts to reduce the 'potency of adversity' and the linking of 'survival and self-reliance' resonate with the findings of work in Britain amongst marginalized Caribbean mothers (Edge and Rogers 2005).

\section{Birth experiences in Burma and during displacement}

Maternal and child mortality is high under the conditions that the Karen women experienced prior to resettlement (Mullany et al 2008, 1689; 1693). The maternal mortality rate in Burma is around 380 maternal deaths per 100, 000 live births (WHO 2007) although in the conflict zones maternal mortality is estimated at 1000 per 100,000 live births (Mullany et al 2008, 1690). According to a survey of recent births, 85\% of ethnic minority women in Eastern Burma reported home births and only 5.1\% recorded the presence of a skilled attendant at the birth (Mullany et al 2008, 1689). 
Prior to arrival to Australia, the women in this study experienced active, natural births with little pain relief, either at home in the village or refugee camp or in a relatively informal, low-resourced camp hospital. In women’s accounts, unmanageable complications in Burma often resulted in maternal death. If in the camp, they were transferred to a rural Thai hospital but in several interview accounts mothers and babies died before they arrived at the hospital. In villages and camps, women relied on emotional and physical accompaniment throughout labour by family members and traditional midwives. Women relayed accounts of traditional midwives or birth attendants (TBAs) who touched, massaged, and 'petted' them and could manipulate the baby into the correct birth position and help deliver the baby. Narratives contained many expressions of gratitude for being able to rest after the birth and family, particularly mothers helping, cleaning and cooking for the post-partum mother.

Many women recounted traditional medicines, foods, treatments and customary practices they believed maintained good health and well-being during the birth and perinatal period which were absent in Australian hospital settings and which they implemented on their return home. Karen birthing practices can be seen as variations on post-partum practices of humoral medicine. These ancient beliefs surface throughout South East Asia, including Burma (Manderson 1981, 509-10). They draw on ideas of hot and cold elements and bear similarity to the ideas from traditional Chinese medicine that understands pregnancy and childbirth as leaving women 'in a state of imbalance much like that of illness’ (Pillsbury 1978).

Many interview accounts referred to principles post-partum women must abide by: staying warm, being protected from cold and wind, not using cold water, eating certain foods, and undertaking certain practices to correct a bodily imbalance and heating up the body. Several women mentioned not washing hair and not getting the 
head cold. Women spoke about making sure they stayed warm after birth, sitting by the fire or heater and taking a steam bath or rubbing certain herbs on their skin to warm them. They advocated using a hot brick after birth to sit on or put on the abdomen to ensure blood did not congeal inside the uterus or as an aid to getting rid of 'bad' blood or 'dissolving' blood so that it could be expelled (these treatments are described in Burma in Manderson 1981, 514-16).

Most women spoke about diet, the consumption and use of turmeric on the body, and Burmese herbs to promote beneficial sweating. Many mentioned not eating cold food, chili, certain strong vegetables that could thicken the blood and also red meat (two women mentioned deer meat causing miscarriage). Women related that not observing these requirements might lead to serious illness in later life.

\section{Birth experiences in Australia: narratives of gratitude, acceptance, confusion}

\section{and complaint}

Women's narratives of childbirth in Australia were dominated by experiences within public hospitals and in most accounts, no interpreters were present. Exact events were often obscured by substantial levels of confusion and misunderstanding due to low levels of communication. Only two women referred to giving written consent for medical procedures and anxiety often resulted from unfamiliar procedures that had not been explained. Confusion and misunderstanding such as that described by 38year-old mother of five, Yee were common: 'I went to hospital when I was pregnant and they checked my baby using a computer. I gave birth and still didn't know and understand anything (laughing). I will tell you, I don't understand a thing.' While Yee presented her confusion as benign, others, such as Baw below, found this experience 'hard'. Many participants described physical complications during and after delivery 
(Ma Sha; Say Say; Ruth; Nin; Hsa; Wah; Rosy) which are presented below. Physical difficulties were exacerbated by a lack of understanding of medical procedures and poor communication between patient and provider.

Most women related confusion over unfamiliar hospital procedures such fetal monitoring, induction, epidurals and vacuum delivery and were unaware of some basic biomedical principles, including hormonal activity and causes and implications of post-natal bleeding, which are the foundation of these medical practices. It was not always clear, if women simply did not understand or if they were contesting institutional narratives in Australia (Barclay and Kent 1998,8; Griffith 2010).

\section{Normalising distress, self-reliance and 'gracious acceptance'}

Baw, a 29-year-old Karen mother with a personal history of loss, had previously borne four children in a refugee camp. Her husband was absent for several of the births and she described pride in her self-reliance in supporting her family. Her account of her fifth birth in Australia demonstrates that even when physical problems are negligible, labor can become distressing due to the lack of familiar social supports and being unable to communicate with those assisting with the labor. Baw compared her treatment in an Australian hospital favorably to the unsympathetic treatment in the refugee camp hospital saying that Australian nurses, ' ...looked after us very well.... those nurses have a good heart and good intention[s].' As she was not able to communicate with the nurses, her assessment of their helpfulness relied on her feelings about their 'good heart' and trust in their 'good intentions' that might be associated with her overall gratitude to the provision of asylum in Australia.

To avoid complications that might arise due to the impossibility of communicating verbally, Baw attended the hospital fully prepared with all the items she would need: 
It is so hard to go to hospital when you are in labor because we cannot communicate... I prepared everything and brought it with me because I didn’t want to make more work for the nurses and I couldn’t tell them anything. I put everything I would need in my bag and when they asked me if I needed anything, I showed them the things in my bag. They asked me if I could understand everything, they said but I didn’t understand anything. I can’t speak English, so it's not easy in this country. It's very hard.

Baw appears relatively content with her treatment in the hospital and located her difficulties within the context of living in a country where she did not understand the language and was scared to speak to people, indicating a sense of resigned displacement. Poignantly at the end of the interview she said that she was 'happy to give birth among [her] own people' in the refugee camp. Thus, even though the conditions were less medically safe than Australia, her emotional experience amongst 'her people’ was more satisfying. Her language indicates a distancing of herself from her new environment, while mourning the loss of an intimate, empathetic community. Say Say, a 43-year-old mother of eight children aged from 21 to 16-month-old twins, described the physical distress of delivering twins by caesarian section in an Australian hospital followed by a painful three-month recovery. Her reaction to the hospital reveals an implicit trust in the sophisticated services. Previously a nurse in the Karen revolutionary movement, Say Say noted that she would never nurse again because the profession in Australian was so much more advanced and she'd never be able to 'catch up'. She perceived a system more sophisticated than anything she had experienced and this might explain her uncritical acceptance of a longer and more painful convalescence than expected. In such a view, staff are beyond reproach and she allows herself no basis from which to question or advocate for her own treatment. 
Combined with this was her attitude to adversity forged through previous births in exceptionally difficult conditions inside Burma with no shelter or medical assistance. Her third and forth babies were born in extreme circumstances while fleeing attacks by the Burmese army and she describes her life then as 'in God's hands', recalling the old Burmese adage mentioned above about women in labor existing somewhere between life and death. Speaking of her slow, painful convalescence post-caesarean in Australia she called upon this old stoicism: 'I suffered the pain from the wound. My husband helped me walk but with other things, I didn’t need his help. I am self-reliant and have depended on myself before...' Extreme hardship had become normal for her and provided an imperative to self-reliance and survival. Her account of self-reliance and stoicism mitigated against Say Say being critical of her treatment in Australia. Ruth, a 40-year-old mother of six, describes an emergency induction of premature twins soon after her arrival in Australia. Her first pre-natal examination revealed health concerns for one of the twins in utero. During delivery, she related that it was communicated to her that her son, '[d]id not have a head', in reaction to which she recalls: 'My heart was shaking.' The delivery was successful; although her son required intensive care, he survived and thrived. During her week in hospital, she said: 'I didn’t even have an interpreter. I just used my hands and legs... I don’t understand what people say to me [and] I usually respond in Karen language and [the nurse] responds to me in English’ Two years later Ruth still did not fully understand why or how the medical procedures were performed; and confusion and distress over this is evident in her account. Ruth had no agency to give consent, advocate for herself or negotiate treatment. While the emergency nature of the intervention may have initially lead to the lack of explanation of medical procedures, that this has remained unaddressed compounds the disempowerment. 
Like Baw, Ruth is grateful for how the nurses looked after her. The family understood that the baby only survived due to the treatment they received in Australia. Ruth's 'gracious acceptance' of the saving of her son's life mitigates against criticism for the lack of consideration she received during delivery and afterwards.

Ma Sha, a 35 year old mother of six, gave the most distressing account of an inpatient stay and demonstrates a deep and unmediated disjuncture between her perspective and that of the health service she attended, corroborating the 'collision of two cultures’ described by Fadiman 1997 amongst the Hmong and their American doctors. Ma Sha described contacting her local hospital for the first time only after labor commenced and encountered a delayed admission process while the hospital checked if they could accommodate her. The hospital could not have known that her personal history had been particularly traumatic marked by series of tragic losses followed by poor conditions in a refugee camp in Thailand where she received a brief education and was married at the age of 16 . Two days before the birth of her third child the camp was attacked and destroyed. Ma Shah described a difficult birth in the open, which was followed by months of profound postnatal emotional distress including frightening hallucinations, which she said, had returned after every subsequent birth.

Ma Sha’s unscheduled admission to hospital in Australia meant no medical or social history existed. The account of her labor illustrates her severe distress:

I wanted to move and walk but they didn’t allow it. I was annoyed but couldn't do anything and didn’t say anything because I knew they couldn’t understand me. There was no interpreter. A few minutes later, two nurses came and tied me up and I could not move. I was scared and thought: 'Something's wrong now,' 
and 'That's it. That's the end of everything'. I felt like I was in a place where people are slaughtered.

Due to the lack of interpreting services available Ma Sha was unable tell hospital staff about her post-natal anxiety and the return of the frightening hallucinations. Therefore due to Mah Shah's initial negative experience, caused largely by the absence of interpreters and communication, she was reticence to confide in staff about her needs or her illness or to make any revelations that might increase her distressing hospital stay and what she understood was an imposed separation from her family who were her main carers. The initial lack of services reduced any possibility of alleviating Ma Sha's anxiety or attention to her possible mental health issues which remain untreated. The gap in communication between Australian medical professionals and patients also reduced the potential for any cultural understanding of Ma Shah's expectations to be able to move around freely and be accompanied through labour by a midwife and her family and not to be left alone during and after labor. Due to her recent arrival in Australia and her unfamiliarity with Australian health service processes and procedures it difficult to objectively conclude actual events and Ma Sha's account is mediated by these limitations. While different explanations for events described by Ma Sha's may be possible with the input of medical staff, our point is that her account is valid for revealing a recent migrant's experience and the multiple effects of not being able to communicate with staff.

Ma Sha's lack of complaint and resignation to receiving treatment causing distress may result from her past experiences and low expectations of entitlement to good treatment. The provision of an interpreter might have avoided some, if not all, of the distress of hospitalisation for Ma Sha. Describing herself as a 'bit down' she noted 
she did not feel part of the Australian community; and the only benefit of being in Australia was that her children would have a better life.

All the women above indicate gratitude for their safe haven in Australia (if only for their children's sake) and acceptance of the hospital treatment without obvious complaint. This can be explained partly by lack of knowledge about what the services should provide (such as interpreting services) and fundamental confusion and miscommunication while in-patients. The 'gracious acceptance' stance adopted by some forced migrants was apparent. This may be the result of imperatives to normalize distress necessitated by the extremely adverse past experiences and their self-reliant attitudes.

There also maybe cultural explanations for not complaining that are hinted at in an account by Nin below. Generally, women did not talk about their emotions and this may be explained by the imperatives to normalize distress outlined above and also feelings of alterity or inferiority and the cultural notion of kaw tha (the disturbed heart): a state marked by difficulty in expressing desires or negative feelings.

\section{Complaint, discrimination and entitlement}

While the previous narratives above are not without implicit or muted criticism, this is much more explicit in the accounts to follow. In the narratives below of younger women, less traumatic personal histories were presented. Raised primarily in refugee camps, most completed high school, including, English language training and had gone on to do voluntary work in schools or other organizations within the camp. Hsa mentioned earlier, volunteered with a medical service that crossed into Burma to treat those in need. Hence, younger refugees had not just much more education, training and employment experience, they also had proportionally more exposure to a 
discourse of human rights and obligations even if this was seldom fulfilled. Due to their age, younger women had experienced a lesser proportion of their lives in conflict zones where adversity was extreme, and a larger proportion of their lives in refugee camps where some measure of protection was afforded and attempts to implement international norms of human rights were explicit, tied to greater access to health and education services. Mostly, they had continued study and work in Australia and therefore had much better English language proficiency. They better understood the obligations of the health-care services and their own entitlements making them more able to assess their treatment.

Nin, a 26-year-old mother of four, recounted a painful experience of postnatal infection in Australia. She blames the attending doctor for causing the infection which has led to ongoing physical discomfort. While Nin fits the category of the younger mothers who showed greater inclination to complain about their experiences in Australian hospitals she also recounts years of persecution and displacement more akin to accounts of the older mothers. However, Nin arrived at a refugee camp in Thailand when she was eight so did not undergo birth in Burma in difficult circumstances nor did she describe fleeing military attacks which were the attributes of the older mothers in the case studies above. She was receiving psychological counseling and treatment and had not normalized her distress in the same way the older mothers appeared to have.

Nin believes the 'Chinese' doctor who delivered her last baby in Australia caused her infection due to negligence:

I think the hospital staff cut the placenta and parts of it were accidently left inside. We didn't get careful treatment but we can’t do much about it. We have to suffer when we give birth... I feel like that doctor did not look after me very 
well but the nurses were better... He always seems cross and never smiles. I am not sure if it is because we are refugees that they don't treat us very well.... I didn’t say anything and I have to thank him for helping me give birth. However, I could not figure out why they do this to me and why they left these things inside me without checking. ...Maybe he looks down on us because we can’t speak the language.

While Nin believes the doctor treated her and other refugee mothers with discrimination and a lack of care she has taken no action: 'I don't want to sue the doctor. I always remember how people have helped us since we came to Australia... I'm only telling you because you came to me ...my wish is for you to understand our suffering both in the camp and here.' Nin felt beholden to the Australian system and therefore not in a position to complain formally and instead used the interview as a vehicle of informal complaint. She also described Karen people's reticence to advocate for themselves rendering them voiceless (cf. Grove and Zwi 2006, 1939). Wah is a 31-year-old woman who like Nin fled from Burma as a child with her family. The Burmese army subsequently destroyed the first refugee camp they reached and the family fled again to another one in Thailand although she did not dwell on these facts. She finished school and worked to support her family. Since arriving in Australia in 2007, she has lived with her husband, close to extended family. She gained a tertiary Diploma and described her English Language Proficiency as intermediate. While working during her pregnancy she felt very supported by her Australian work colleagues. She gave birth at a local hospital and her account resonates with sensitivity to perceived criticisms about her abilities to feed her child:

The midwives on duty were not so nice. They told me to feed the baby but the problem was I didn’t know how. When we tried it together, they held the head 
of the baby so tightly I was worried for my daughter. ... Then they told me that if the baby was not feeding well I couldn't leave the hospital. ... [The midwife] talked very nicely to the white lady beside me and helped her but she didn’t talk to me like that. I felt like she looked down on me and thought I didn’t understand things so she didn’t treat me well but I'm not sure. I felt like it was discrimination but I might be wrong. She supported the white lady a lot but every time she saw me she would say that everything I did was wrong and complained that I was not learning. I was so disappointed with myself. There wasn’t anyone around to help and my family were only allowed to stay until 8 pm so I had to do everything myself.

Wah felt her outsider status and internalized the perceived ill-treatment by the midwife. She keenly felt the separation from her family who would have provided the care for herself and her baby that she expected and was absent in the hospital.

Rosy was 25 and a sole-parent with a one month old baby boy. Due to the immediacy and emotional impact of the traumatic birth of her son she did not feel confident enough to engage in a face-to-face interview and instead volunteered to record her story and post the file to the research assistant. She had suffered severe distress throughout her unplanned pregnancy because her husband and father of her child was unable to join her in Australia due to immigration requirements. She described a distressing emergency caesarian followed several days later by surgery to overcome an infection.

[They asked if I needed to talk about the post-natal surgery] I said 'Yes', and they organized an interpreter for me. As soon as I arrived the doctor that performed the caesarean asked why I wanted an interpreter now because before [during the caesarean] I had said I didn't need one. She wouldn’t look at me and 
her reaction was strange. I kept asking the same question: 'Why do I have to have the second operation?' ...Suddenly, she didn't look like the same doctor that smiled at me when I was in labor. I couldn't believe my eyes. She told me that when I was trying to push during labor blood had pooled inside me which they didn't see. She... sounded like she was blaming me for pushing too forcefully. I told her that I felt sad about this and asked her how many caesareans I could have. She said she wouldn't encourage me to have a lot of children. Her comment hurt me so much! I told them that I wasn't asking these questions because I was mad with them but because I didn't want to have the second operation because I didn't have anyone to take care of me afterward. The doctor said, 'Sorry', and I said it is fine because without her I might have died. Rosy added later in her account that she did not think the doctor would have 'treated white people in the same way', identifying the treatment as discriminatory.

Nin, Rosy and Wah all described feeling discriminated against while inpatients during confinement but they were all loathe to complain. Nin was the only one who explicitly stated that she felt beholden to the Australian system and did not feel she had the right to complain.

Hsa is a 28-year-old Karen mother of one, who was born and lived in a refugee camp until she was 23. When she finished high school, she carried out volunteer work with a medical NGO in the camp but seeing limited opportunities she migrated to Australia with her sister in 2006. Most of her extended family has followed. In Australia, she studied and gained a tertiary Certificate. She expressed some dissatisfaction with the hospital service during her pregnancy and birth of her daughter and also with her own language proficiency. Her account provides insight into the discrimination she felt and misunderstandings between herself and the hospital: 
The hospital was very good but there was one thing that I didn’t feel good about when I had my baby. Services appear comprehensive in the hospital but when I was in labour and didn’t have strength to walk, I had to catch a lift to get to my bed without any help. I couldn’t walk anymore so I just stood still. People saw me but nobody helped so I just started crying. They simply told me where to go and pointed. I thought I needed a wheelchair but no-one got me one. I cried a lot but they just encouraged me to go by myself. I tried hard and walked to the birthing room and gave birth. After that, a midwife came and helped wrap-up my baby but I think she did it too harshly. The baby was crying and she picked her up, wrapped her, and put her back and left. She didn't say a word to me. I told my husband that I wasn’t happy.

It is hard to understand why no one would help Hsa with a wheelchair or a nurse would not speak to a mother while attending to her newborn baby and it is not known if these were normal behaviors or procedures or if Hsa was being discriminated against. The context of the relationship between patient and health-care provider requires deeper scrutiny than we can make here. The point here is to describe how Hsa experienced and felt about the service.

Several of the accounts like Wah's and Hsa's illustrate surprise about how little personal care they received from hospital staff and compounding this how family were not permitted to stay and care for them either. Women expected to be accompanied and cared for through labor and perinatally by a mid-wife and family and were frightened to be left alone while in labor with no idea about what would happen next, especially the younger mothers. Most, like the older mothers, Ma Sha in particular, but Wah also, were desperate to get home to the relief of family care and to 
therapeutic food and heat treatments the absence of which in the hospital caused confusion and distress.

The gulf between birthing practices familiar to the women and those carried out in modern Australian hospitals was great. There were many examples of the absence or prohibition by staff of cultural aspects to labor important to women, particularly family involvement, massage, movement and adherence to specific therapeutic treatments. Women described unfamiliar restrictions on their behavior while giving birth as discomforting or upsetting. Even though most praised the attentions of nurses (one saying the nurses looked 'after her like their sister') and were reassured by the superior medical services of Australian hospitals, many indicated a preference to give birth amongst their own family and community where they could communicate in their own language and be assured of familiar comforts. If they did not directly complain about the lack of interpreting services, they all mentioned how difficult this had been for them.

\section{Concluding Remarks}

Many of the Karen women in this study described how self-reliance was the key to survival and provided insight into the different strategies they used to help themselves when they felt emotionally distressed. Some like Ela, advocated a mental regime which involved 'controlling your heart', explaining that she had survived years of hardship in the refugee camps in this way. Of the younger mothers, Hsa had multiple strategies like walking, talking to her family and colleagues. Although Nin was receiving professional psychological treatment to address her distress (presumably through an interpreter), she also had personal strategies: 'I turn the music on, I will listen and sing along.... I find comfort in playing games because I can bury my heart 
there.' Ma Shah received much personal care from her extended family. All of the women interviewed had a fundamental belief in God and many of the women found great solace in spirituality, both Christian and Buddhist. Similarly to Edge’s study, it appears that Karen women are likely to draw on spirituality for emotional support and that 'spirituality was a powerful weapon for dealing with adversity' (Edge and Rogers 2005).

The women in our study were familiar with a paradigm of active, natural birthing within a community setting and were unsettled, confused, bewildered and sometimes frightened by the vastly different biomedical systems and settings of births in Australia. Australia is criticized for having one of the highest rates of medical intervention into birth in the world (Donnellan-Fernandez 2010) and this is one of huge differences faced by the women in this study. The large Australian hospitals the women and their families encountered for the first time were not only physically alienating but required they navigate relations with professional staff with whom they could not communicate because of lack of interpreting assistance. The most often repeated solution to the difficulties described by women in this study was for more and better translation/interpreting services so they could communicate and have some agency in their health-care. They also spoke fondly and in detail about their own customs relating to perinatal health as described above.

Similar situations and lack of translation services are described in other settings around the world (for UK see Griffith 2010 and for Canada see Anderson et al 2003). The complicated effort of cross cultural communications and interpretation goes both ways as Fadiman suggests. The Canadian study notes: 'through language people are able to negotiate meanings and come to shared understandings' and 'people's ability to communicate with one another is fundamental to a feeling of safety’ (Anderson et 
al 2003, 209). The women in our study often felt unsafe and this prompts us to reiterate the questions posed by Anderson et al (2003) about whether there might not be an expectation amongst health-care staff, 'that patients ought, in fact, to be able to communicate in English?' Our research highlights the need for more interpreting services.

The women's reactions to the health services they received varied along gradations of gratitude, acceptance, confusion, misunderstanding, incomprehension, complaint and feelings of discrimination depending on age and social and medical histories. Younger women appeared more willing to complain and identify discrimination. This difference is traced in women's reactions to treatment and services with respect to those who were accepting to those who felt entitled to complain and why. We found that women's accounts in our study were located across a continuum of reactions: from overwhelming gratitude to a preparedness to voice complaint about the obstetric care they received in Australian hospitals and their position on this continuum related strongly to their pre-settlement experiences. Yet, 'explanations that place the onus on the culture of the individual are likely to lead to individual-centered interventions at the expense of addressing the structural contexts that reproduce social and economic inequities’ (Viruell-Fuentes , Mirada and Abdulrahmin 2012, 5).

Nowhere are these inequities more obvious than in the lack of interpreting services offered to the mothers in this study. Our data reveals fully the impact this has had on their health-care experiences. We suggest therefore that the power relations inherent in the interactions between patient and health-care provider in this regard need to be scrutinized with, 'an appreciation of both our postcolonial historical heritage and the present context in which health care is delivered.' Further research with health care providers could complete this picture and needs to be pursued. This research provides 
insights into the experiences of the health care system of Karen women in Australia, through a focus on their experiences of childbirth. We focus on narratives of gratitude and complaint in this analysis to highlight the complex differences in women's experiences, their own agency and expectations of, and sense of entitlement to good care in the Australian health system. Entitlement to care-and an expectation of receiving good care and the right to complain if care is not so-serve, amongst other things, as a marker of a sense of belonging, of inclusion, and of acceptance. The entitlement of forced migrants to social services, including health care services, remains a controversial and divisive issue in many host societies, Australia included. Based on women's accounts we highlight the need for sensitive and inclusive professional medical services and reiterate women's explicit request for increased interpreting services.

Our findings also point to challenges beyond health services. Australian popular discourse continues to frame providing asylum as a charitable concession rather than an obligation to which forced migrants are entitled, and continues to see forced migrants as 'overloading' social systems designed for others more entitled than they. In this environment the systemic issues related to appropriate health care and other services to immigrants will continue to be an issue as will over-determined discourses of deservingness (and non-deservingness) and gratitude which risk perpetuating the inequities and disadvantage which marks the lives of forced migrants and provision of services for asylum seekers. This is a vexed political issue in Australia, as in many other countries. The problems with health care provided to the women in this study coupled with the inability of many of the women to voice complaints about this care are problems, which await address not simply within the health-care system, but a 
wider political and social address of the resentment, which surrounds Australia's obligation to those who seek asylum within its borders.

\section{References}

Anderson, Joan, JoAnn Perry, Connie Blue, Annette Browne, Angela Henderson, Koushambhi Basu Khan, Sheryl Reimer Kirkham, Judith Lynam, Pat Semeniuk and Vicki Smye 2003 “Rewriting” Cultural Safety Within the Postcolonial and Postnational Feminist Project, Toward New Epistemologies of Healing. Advances in Nursing Science 26(3): 196-214.

Barclay, Lesley and Diane Kent 1998 Recent immigration and the misery of motherhood: a discussion of pertinent issues. Midwifery 14: 4-9.

Bhabha, Homi. K.

1994 The Location of Culture, Routledge.

Cartwright, E and Lenore Manderson

2011 Editorial: Diagnosing the Structure: Immigrant Vulnerabilities in Global Perspective. Medical Anthropology: Cross-Cultural Studies in Health and Illness 30(5): 451-453.

Connell, Raewyn

2012 Gender, health and theory: Conceptualizing the issue, in local and world perspective. Social Science and Medicine 74:1675-1683.

Donnellan-Fernandez, Roslyn

2010 Having a Baby in Australia: Women's Business, Risky Business, or Big Business? Outskirts Online Journal 24. 
http://www.outskirts.arts.uwa.edu.au/volumes/volume-24/donnellan-fernandez, accessed May 2012.

Dossa, Parin

2002 Narrative mediation of conventional and new 'mental health' paradigms:

Reading the stories of immigrant Iranian women. Medical Anthropology Quarterly 16(3): 341-359.

Edge, Dawn and Anne Rogers

2005 'Dealing with It’: Black Caribbean Women's Response to Adversity and Psychological Distress Associated with Pregnancy, Childbirth, and Early Motherhood. Social Science and Medicine 61(1): 15-25.

Fadiman, Anne

1997 The spirit catches you and you fall down: A Hmong child, her American doctors, and the collision of two cultures. New York: Farrar Straus and Giroux.

Griffith, Laura

2010 Motherhood, ethnicity and experience: a narrative analysis of the debates concerning culture in the provision of health services for Bangladeshi mothers in East London. Anthropology and Medicine 17(3): 289-299.

Grove, Natalie. J. and Anthony. B. Zwi

2006 Our health and theirs: Forced migration, othering, and public health. Social Science and Medicine 62:1931-1942.

Harkins Benjamin

2012 Beyond “Temporary Shelter”: A Case Study of Karen Refugee Resettlement in St. Paul, Minnesota, Journal of Immigrant \& Refugee Studies, 10:2, 184-203.

Killingsworth, Ben, Renata Kokanovic, Huong Tran, and Chris Dowrick 
2010 A Care-Full diagnosis: Three Vietnamese-Australian Women and their experience of becoming “mentally ill”. Medical Anthropology Quarterly. 4(1): 108-123.

Kokanovic Renata, Christopher Dowrick, Ella Butler, Helen Herrman and Jane Gunn 2008 Lay accounts of depression amongst Anglo-Australian residents and East African refugees. Social Sciences and Medicine 66(30): 454-466.

Kokanovic Renata, John Furler, Carl May, Christopher Dowrick, Helen Herrman, Helen Evert and Jane Gunn

2009 The politics of conducting research on depression in cross-cultural context. Qualitative Health Research 19(5): 708-717.

Kokanovic, Renata and Meredith Stone

2010 Doctors and other dangers: Narratives of distress and exile in Bosnian refugees in Australia. Social Theory and Health. 8(4): 350-369.

Kokanovic, Renata, Carl May, Christopher Dowrick, John Furler, Danielle Newton and Jane Gunn

2010 Negotiating distress between East Timorese and Vietnamese migrants in Melbourne and their family doctors. Sociology of Health and Illness, 32(4):511527.

Kokanovic, Renata, Sara L. Niner., Violet Cho and Denise Cuthbert 2012 Pilot Study on perinatal depression (PD) in refugee communities. Research report prepared for beyond blue. Melbourne.

Manderson, Lenore

1981 Roasting, smoking and dieting in response to birth: Malay confinement in cross-cultural perspective. Social Science and Medicine 15B: 509-20.

Manderson, Lenore and Pascale Allotey 
2003 Storytelling, marginality, and community in Australia: How immigrants position their difference in health care settings. Medical Anthropology: CrossCultural Studies in Health and Illness 22(1): 1-21.

McKay, Fiona H., Samantha L. Thomas, and Susan Kneebone 2011 'It Would be Okay If They Came through the Proper Channels': Community Perceptions and Attitudes toward Asylum Seekers in Australia'. Journal of Refugee Studies 25(1): 113-133.

Mullany, Luke C., Catherine I. Lee, Lin Yone, Palae Paw, Eh Kalu Shwe Oo, Cynthia Maung, and Thomas J. Lee

2008 Access to Essential Maternal Health Interventions and Human Rights Violation among Vulnerable Communities in Eastern Burma. PLOS Medicine 5(12): 16891697.

O'Kane, Mary

2005 Borderlands and Women: Transversal Political Agency on the Burma-Thailand Border. Melbourne: Monash Asia Institute.

Oo, Zin Mar. and Koyoko Kusakabe

2010 Motherhood and social network: Response strategies of internally displaced Karen women in Taungoo district. Women's Studies International Forum, $33: 482-491$.

Pillsbury, Barbara L. K.

1978 'Doing the month': confinement and convalescence of Chinese women after childbirth. Social Science and Medicine 12: 11-22.

Quesada, James, Laurie Kain Hart and Philippe Bourgois 2011 Introduction: Structural Vulnerability and Health: Latino Migrant Laborers in the United States. Medical Anthropology: Cross-Cultural Studies in Health and Illness. 30(4): 339-362. 
Quesada, James

2012 Special Issue Part II: Commentary Illegalization and Embodied Vulnerability in Health. Social Science and Medicine 74(6): 894-896.

Sargent, Carolyn

2012 'Deservingness’ and the politics of health care, Theme Issue Part I, Social Science and Medicine 74(6): 855-857.

Tummala-Narra, Pratyusha

2004 Mothering in a Foreign Land. American Journal of Psychoanalysis 64(2):167182.

Viruell-Fuentes, Edna A., Patricia Y. Miranda and Sawsan Abdulrahim 2012 (In Press) Review Article: More than culture: Structural racism, intersectionality theory, and immigrant health. Social Science and Medicine http://dx.doi.org/10.1016/j.socscimed.2011.12.037, accessed August 2012 Willen, Sarah S.

2012 Introduction Special Issue: Migration, “illegality,” and health: Mapping embodied vulnerability and debating health-related deservingness. Social Science and Medicine 74(6): 805-811.

World Health Organization (WHO)

2007 Part 1: Report Millennium Development Goals. http://www.who.int/whosis/whostat/EN_WHS09_Part1.pdf, accessed August 2012 